\title{
Jacobi contra Fichte, el Mesías de la razón pura
}

\section{María Jimena Solé}

\section{OpenEdition}

\section{Journals}

Edición electrónica

URL: http://journals.openedition.org/ref/332

DOI: $10.4000 /$ ref.332

ISSN: 2258-014X

Editor

EuroPhilosophie Editions

Referencia electrónica

María Jimena Solé, "Jacobi contra Fichte, el Mesías de la razón pura », Revista de Estud(i)os sobre Fichte [En línea], 1 | 2010, Publicado el 14 junio 2010, consultado el 08 septiembre 2020. URL : http:// journals.openedition.org/ref/332 ; DOI : https://doi.org/10.4000/ref.332

Este documento fue generado automáticamente el 8 septiembre 2020.

(c) EuroPhilosophie 


\title{
Jacobi contra Fichte, el Mesías de la razón pura
}

\author{
María Jimena Solé
}

\section{NOTA DEL AUTOR}

Este texto es una versión preliminar de mi artículo "La intervención de F. H. Jacobi en la Disputa sobre el ateísmo" publicado en: J. Rivera de Rosales y Óscar Cubo (eds.) La polémica del ateísmo. Fichte y su época. Madrid 2009: 449-462. Salvo que se indique lo contrario, la traducción al español de los textos de Fichte y de Jacobi es mía.

1 En noviembre de 1798 el príncipe elector de Sajonia ordena la confiscación de los dos primeros artículos del octavo volumen del Philosophisches Journal, el periódico filosófico que F. I. Niethammer y Fichte editaban en conjunto, por contener, a su entender, expresiones ateas. Como se sabe, el edicto hacía referencia al escrito titulado Desarrollo del concepto de religión de Karl Forberg y al artículo redactado por Fichte - como un comentario crítico a éste - Sobre el fundamento de nuestra creencia en un gobierno divino del mundo. Con diferentes matices, una idea claramente inaceptable para las autoridades políticas y eclesiásticas atravesaba estos dos textos: la primacía de la moral sobre la religión. A principios de enero de 1799 Fichte reacciona frente a esta situación mediante la publicación de su Apelación al público (AP), en la que se defiende de la acusación de ateísmo que las autoridades habían dejado caer sobre él. Luego de señalar en este escrito que la denuncia era una excusa y que la persecución en su contra respondía a otros motivos, (cf. GA I, 5, 421 ss. AP) y de presentar nuevamente su concepción de Dios como el orden moral, ya desarrollada en su artículo prohibido por el príncipe elector de Sajonia, Fichte hace referencia a tres hombres que, libres de toda sospecha, compartirían con él sus convicciones: el teólogo J. J. Spalding, el primer predicador de la corte de Dresden, F. V. Reinhard y, finalmente, "entre los filósofos", apela al "noble Jacobi" (edler Jacobi), cuya mano dice tomar con toda confianza. "Aún cuando podamos pensar diferente acerca de la pura teoría", declara Fichte, "de lo que aquí se trata, tú ya lo has dicho hace mucho tiempo, tal como yo lo pienso; lo has dicho 
con una fuerza y un calor, con los que yo nunca podré expresarlo, y lo has convertido en el espíritu de tu filosofía: «por medio de una vida divina el hombre se vuelve uno con Dios »." (GA I, 5, 447 AP)

Pocas semanas después de recibir un ejemplar de la Apelación Jacobi responde en su famosa Carta a Fichte (Brief an Fichte), ${ }^{1}$ en la que pesar de presentarse como su defensor, es incapaz de ofrecer la ayuda que el acusado esperaba. Jacobi no sólo desestima la posibilidad de una coincidencia entre su punto de vista y el fichteano, sino que además critica la Doctrina de la Ciencia entendiéndola como la radicalización del proyecto racionalista de la Ilustración ya denunciado por Jacobi en su correspondencia con Mendelssohn como una marcha segura hacia el ateísmo, el fatalismo y el nihilismo. (cf. JW IV.1, 59 ss.)

3 El objetivo de este trabajo es analizar la crítica de Jacobi a Fichte tal como aparece en su Carta de 1799. Para esto nos detendremos previamente en la relación que existía entre ambos pensadores con el fin de intentar comprender por qué Fichte cree haber encontrado un aliado en este enemigo de la Ilustración. Luego expondremos la interpretación que hace Jacobi de la Doctrina de la Ciencia como el sistema más acabado de la filosofía racionalista - cargo por el cual Jacobi bautiza a Fichte como el Mesías de la razón especulativa -. Para finalizar, nos detendremos brevemente en la reacción de Fichte frente a estas críticas a su sistema.

\section{Jacobi y Fichte}

La confiada apelación de Fichte a Jacobi parece inexplicable, si tenemos en cuenta que algunos años antes Jacobi había exhortado a Lessing nada menos que a abandonar la razón mediante un salto mortal a la fe. (cf. JW IV.1, 59) Sin embargo, no faltan pruebas de la gran admiración que el autor de la Doctrina de la Ciencia sentía por el polémico detractor de la filosofía racionalista. "Si existe un pensador en Alemania con el que deseo y espero coincidir en mis convicciones particulares, ese es usted", (GA III, 2, 202) le confiesa en su primera carta de 1794.

5 Un año más tarde Fichte vuelve a dirigirse a Jacobi, para informarle que al releer sus escritos, ha sido sorprendido, especialmente en la novela Allwill ${ }^{3}$, al comprobar la evidente coincidencia entre las convicciones filosóficas de ambos (cf. GA III, 2, 391). Sin embargo, Fichte encuentra una diferencia entre ellos: "Como es sabido", escribe, "usted es realista y yo soy idealista trascendental, más riguroso aún que Kant, pues en él existe todavía la diversidad de la experiencia; por el contrario [...] yo supongo que incluso ésta es producida por nosotros mediante una capacidad creadora". (ibíd.) Fichte debió suponer que Jacobi estaría satisfecho al comprobar que él había adoptado su famosa crítica a la filosofía kantiana: la imposibilidad de ingresar al sistema sin la cosa en sí y la imposibilidad de mantenerse en él admitiendo la existencia de cosas en sí. (cf. JW II, 291 ss. $)^{4}$

6 Pero Jacobi no celebró este triunfo. Tampoco pareció prestar mayor atención al hecho de que, como le aseguraba Fichte en una tercera carta de 1796, ambos buscaban la verdad en el mismo sitio, a saber: "en el santuario más interior del propio ser." (GA III, $3,18)$ La única diferencia entre ellos era, según Fichte, que mientras Jacobi lograba revelar al espíritu en cuanto espíritu, él se había propuesto la tarea de interpretarlo bajo la forma de un sistema. 
7 La primera reacción por parte de Jacobi hacia Fichte, después de leer Sobre el concepto de la Doctrina de la ciencia (BWL) que Goethe le había hecho llegar en mayo de 1794, fue positiva. (cf. JABr II, 173) ${ }^{5}$ Pero sabemos por su demorada respuesta a las dos primeras cartas de Fichte - de diciembre de 1795 - que en esa fecha aún no había estudiado las obras que él le había enviado. Sin embargo, allí mismo dice tener la intención de hacerlo pronto, para poder comunicarle su opinión acerca de la armonía entre ellos. (cf. $\mathrm{JABr}$ II, 214-215) Al año siguiente, recibe la tercera carta de Fichte y no la responde.

8 Si bien no hay indicios de que Jacobi se haya ocupado seriamente de Fichte antes del estallido de la Disputa sobre el ateísmo, sabemos gracias a los así llamados Denkbücher -un conjunto de trece cuadernos de anotaciones hechas por Jacobi entre los años $1787 \mathrm{y}$ 1819, de los cuales hoy faltan los cuadernos III y XI- ${ }^{6}$ que luego de recibir la Apelación al público Jacobi se abocó seriamente y con suma intensidad a su estudio. En efecto, en los Denkbücher Jacobi confiesa haber dedicado dos de sus cuadernos borradores de trabajo, una serie paralela de anotaciones a sus Denkbücher, a la preparación de la famosa carta a Fichte, ${ }^{7}$ algo que, según I. Radrizzani, Jacobi "no hizo por ningún otro filósofo". ${ }^{8}$ Este esfuerzo se ve reflejado además en las referencias que encontramos en la carta a diferentes obras de Fichte que, sabemos, Jacobi poseía en su biblioteca. ${ }^{9}$

El 18 de enero de 1799 Jacobi recibe de Fichte una copia de su Apelación al público con la siguiente nota: "De usted no espero ni una muestra de interés, ni una intervención, ni nada por el estilo, busco solamente amistad." (GA III, 3, 176) Entre el 3 y el 21 de marzo redacta su Carta a Fichte en la que no sólo no defiende al acusado del cargo de ateísmo, sino que agrega una nueva acusación: según Jacobi Fichte es el Mesías de la razón especulativa pues lleva la pretensión humana de explicarlo todo aún más lejos que Kant - a quien se refiere como el Bautista de Königsberg - y que el renombrado ateo Spinoza.

10 Jacobi envió este escrito a Fichte a través de Reinhold y Fichte respondió desde Jena el 22 de abril, agradeciendo sinceramente el "excelente escrito" (das treffliche Schreiben) en su favor (für mich) y admitiendo que los últimos acontecimientos no le habían permitido tomarse el tiempo de estudiar la carta en profundidad, como para encontrar en qué medida el escrito podía ser considerado en su contra. (cf. GA III, 3, 334) Frente a esta primera reacción positiva de Fichte, Jacobi debe haberse sentido autorizado a pensar en la publicación de su escrito. ${ }^{10}$ Así pues, en otoño de ese mismo año, la carta apareció con el título Jacobi an Fichte, en una versión sustancialmente aumentada por la inclusión de un prefacio, tres apéndices y cinco añadidos. A pesar de que Jacobi aseguraba que las modificaciones habían sido menores, las diferencias entre la versión manuscrita y la dada a la imprenta eran significativas. En efecto, la versión impresa tomaba en consideración las interpretaciones y repercusiones generadas por la primera versión de la carta, que circuló en copias en el mundo académico. ${ }^{11} \mathrm{El}$ escrito tuvo una fuerte repercusión y generó opiniones que alimentaron la polémica.

\section{Fichte o la promesa de una filosofía pura}

11 Quien está familiarizado con la obra de Jacobi, especialmente con los textos de la denominada Polémica del panteísmo, no puede dejar de advertir una clara continuidad entre ellos y la Carta a Fichte. En efecto, el núcleo central de la crítica al sistema fictheano en esta carta - la interpretación de la Doctrina de la ciencia como un spinozismo invertido y, por lo tanto, como un ateísmo y un nihilismo - se apoya sobre su controvertida lectura de Spinoza presentada en su correspondencia con Mendelssohn 
entre los años 1783 y 1784, que Jacobi recopila y publica en 1785 y nuevamente en 1789 bajo el título Über die Lehre des Spinoza in Briefen an Herrn Moses Mendelssohn (Cartas al Señor Moses Mendelsohn sobre la doctrina de Spinoza).

Allí Jacobi presenta el sistema spinoziano como la expresión más perfecta y coherente del racionalismo. La opinión de Jacobi se funda en el hecho de que, según él, Spinoza es el único filósofo de la historia que se ha atrevido a explicitar y a aceptar las consecuencias a las que conduce una razón teórica que pretende explicarlo todo. (cf. JW IV. 2, 133) Estas consecuencias, que Jacobi considera suficientes para rechazar la filosofía racionalista en su conjunto, son el fatalismo, el ateísmo, el panteísmo. (cf. JW IV. 1, 56 ss.) Este lugar privilegiado en la historia del pensamiento que Jacobi había reservado hasta ese momento para Spinoza, será ahora, en 1799, ocupado por Fichte. Él se transforma en el nuevo héroe de la filosofía, que al subvertir el sistema spinoziano, lleva la razón humana aún más lejos en lo que Jacobi considera su inminente marcha hacia el abismo.

Por lo tanto, aquí Jacobi no hace más que volver al ataque contra su antiguo enemigo el racionalismo - utilizando la misma estrategia con que había logrado, unos años antes, poner en peligro a toda la Ilustración alemana. ${ }^{12}$ Su estrategia no es otra que denunciar las consecuencias de una filosofía fundada únicamente en la razón especulativa, sin conexión con las exigencias de la vida, con los sentimientos y con la fe. En 1785 el enemigo había sido Mendelssohn y los ilustrados de Berlín. Luego Kant. Ahora, Fichte. Algunos años más tarde será el turno de Schelling. Pero siempre es Spinoza el objeto de crítica; pues, según Jacobi, la razón, llevada a su extremo, conduce necesariamente al spinozismo. (cf. JW IV.1, 53 y ss.)

En 1799, sin embargo, Jacobi reconoce que Fichte ha alcanzado una nueva cumbre en la historia de la filosofía. En esta historia del desarrollo del impulso por conocer innato en el hombre, Fichte se ha elevado más que cualquier otro y se revela como el "el hijo verdadero de la promesa de una filosofía completamente pura, consistente en y por sí misma". (JaF 9 s.) A pesar de los esfuerzos de Fichte, Jacobi ve en él a un pensador que, al igual que todos los que lo precedieron, se limita estrictamente al punto de vista del saber, que permanece dentro de los límites de la razón teórica con la única virtud de ser quien la ha desarrollado con máxima coherencia.

15 Jacobi caracteriza el espíritu de la filosofía racionalista, o filosofía sin más, como el esfuerzo por distinguir dos proposiciones - "yo soy" y "existen las cosas externas a mí" - de cuya idéntica certeza cualquier hombre que persista en su actitud natural jamás dudaría. Una vez diseccionadas, el filósofo intenta deducir una a partir de la otra. Recurriendo a la terminología fichteana, Jacobi identifica este proceder como el intento por reducir, sea el Yo al No-Yo, sea el No-Yo al Yo. (cf. JaF 10) Por eso, Jacobi viene a coincidir con Fichte en que la filosofía puede adoptar sólo dos formas diferentes: el dogmatismo (o materialismo) - si intenta explicar todo a partir del No-Yo, esto es, de la materia - o el idealismo - si reduce toda la realidad a un Yo, es decir, a una inteligencia. ${ }^{13}$ Ambos sistemas persiguen, también como en Fichte, una única meta: encontrar un principio, fundamento y causa de todo lo que existe. (cf. JaF 10)

16 Ahora bien, a diferencia de Fichte, y denunciando el proceder de toda la filosofía racionalista, Jacobi postula que, en vez de excluirse mutuamente, se da entre el materialismo y el idealismo una dialéctica que conduce a su interpenetración: el materialismo está destinado a transformarse en idealismo. Para mostrar esto, Jacobi recurre a Spinoza a quien, según él, le "faltó poco" para convertirse en un idealista. (JaF 11) 

filosófico y hacer de la cara superior, la cara del pensar que llamó objetiva, la cara inferior, la que llamó subjetiva formal, e investigar si éste seguía siendo el mismo." (JaF 11-12) ${ }^{14}$ La inversión propuesta por este experimento filosófico consistiría en hacer de lo fundante - la sustancia en cuanto ser subsistente por sí - lo fundado, y de lo fundado - el pensamiento de esa sustancia- la base sobre la cual reposa el ser. Pero al realizar esta inversión, el sistema se vería totalmente transformado: al girar el sólido cuerpo geométrico, la doctrina de Spinoza perdería su forma y, celebra Jacobi, "se convertiría en una llama pura, ardiendo por sí misma, sin necesidad de un lugar ni de material combustible: ¡idealismo trascendental!" (JaF 12)

El materialismo de Spinoza era ya, en germen, un idealismo al estilo fichteano. Una simple inversión le hubiese revelado ese secreto: que únicamente un Yo puede fundar el ser. Frente al robusto cubo del materialismo, el idealismo es caracterizado como algo sin forma ni materia, sin solidez, sin contenido: un Yo incandescente que se alimenta de su propio interior vacío.

Según Jacobi, Fichte vio claramente esta necesaria supremacía de la actividad del pensar. La Doctrina de la Ciencia ha hecho de la sustancia un Yo, ha puesto a Spinoza patas para arriba y, revelándose como un "materialismo sin materia" (JaF 12), lo ha superado.

21 Por eso Jacobi proclama a Fichte como el verdadero Mesías de la razón especulativa. Su signo es "la reunificación del materialismo e idealismo en un ser indivisible". (JaF 14) La Doctrina de la ciencia, al invertir el sistema spinoziano - el materialismo más coherente - supera la oposición entre el ser y el pensar como principios absolutos excluyentes y conquista una forma superior que los engloba. El Yo es a la vez sujeto pensante y materia pensada; "egoísmo", concluye Jacobi. (JaF 11) No hay exterioridad. No hay nada que trascienda al sujeto. El No-Yo se transforma en un momento interior al Yo. Pero tampoco el Yo es algo. Su pureza debe ser total. El Yo existe únicamente en el producir del espíritu: "un mero acto-acto", (Tat-Tat, JaF 20) ironiza Jacobi.

\section{Nihilismo y ateísmo}

Pero esta acción del Yo, que Fichte concibe como productora, es denunciada por Jacobi como un puro aniquilar. (cf. JW IV.1, 398) ${ }^{15} \mathrm{El}$ entendimiento y la imaginación humanas, abstrayendo lo común que se presenta en la experiencia, reflexionando sobre ello, reducen la realidad a mera representación subjetiva. Los objetos pierden su ser independiente y son reemplazados en la mente humana con conceptos y palabras. El mundo exterior es sustituido por una imagen, hecha a la medida de las facultades humanas.

La existencia de las cosas tal como éstas son en sí es, por lo tanto, imposible de conocer. Al querer acceder a ellas mediante conceptos, el filósofo las aniquila, las transforma en nada. Kant y Fichte, reconoce Jacobi, han librado a la humanidad de este sueño de la razón especulativa. (cf. JaF 31) Sin embargo, ellos mismos siguen siendo presas de otra ilusión: la de una razón productora de la verdad, que también conduce al abismo del vacío, al nihilismo. (cf. JaF 44)

"Una filosofía pura, es decir, completamente inmanente [...], sólo es posible a la manera fichteana”, (JaF 19) asegura Jacobi. Este egoísmo absoluto conquistado por Fichte, en el 
que todo se diluye en un Yo que no es sino esa pura acción disolvente, es para Jacobi la forma más acabada y perfecta de la filosofía. No se trata, pues, sólo de un spinozismo invertido sino de un spinozismo perfeccionado. ${ }^{16}$

A esta razón especulativa, productora de la verdad, Jacobi opone otra concepción de la razón humana como una facultad meramente captadora de lo verdadero. (cf. JaF 35) Pues esto, lo verdadero - de lo que, según Jacobi, Fichte "no tiene ninguna noticia" (JaF 17) existe necesariamente fuera de la ciencia del saber, más allá del yo y sus facultades del conocimiento. "Por eso mi lema y mi solución no es el Yo, sino ¡más que Yo! ¡Mejor que Yo! - Algo completamente otro", (JaF 35) exclama Jacobi.

Contra el monismo metafísico del que tanto Fichte como Spinoza son exponentes, Jacobi apela a un dualismo radical: sólo mediante algo que trasciende la mente, el sujeto conquista su individualidad y no se funde, no se pierde a sí mismo en la masa amorfa de lo Absoluto. Ese otro absoluto al que Jacobi apela no es sino el Dios personal y creador, que garantiza no sólo la existencia de cada ser humano como persona individual, como sujeto libre, sino también la existencia del mundo exterior como algo subsistente por sí. $\mathrm{Al}$ idealismo de Fichte, fundado en un Yo productor de un mundo fenoménico, Jacobi opone un realismo basado en la relación inmediata entre un Yo finito y un Tú infinito, trascendente. "El hombre encuentra a Dios porque él puede encontrarse a sí mismo sólo y al mismo tiempo con Dios." (JaF 48) $)^{17}$

Para Jacobi únicamente Dios puede crear algo a partir de la nada. El Yo fichteano, en cambio, en cuanto vacío, sólo crea más nada. Por eso, en la medida en que no reconoce que la existencia de la divinidad trascendente y personal es un una certeza inmediata que se revela en el corazón del hombre, la Doctrina de la ciencia es irremediablemente atea.

Sin embargo, Jacobi considera esto como una torpeza del artista de ideas y palabras (cf. JaF 46). Pues, según él, estrictamente, la filosofía trascendental puede ser tan atea como la matemática pura. Ni la aritmética ni la filosofía saben nada acerca de Dios. Por eso Jacobi reprocha a Fichte haberse ganado la acusación de ateísmo, sin ninguna necesidad. (cf. JaF 7) Fichte nunca debió haber ido más allá de los límites de su razón. Jamás debió pretender que el ser humano podía conocer a Dios mediante sus razonamientos y demostraciones filosóficas.

La única defensa, pues, que Jacobi puede ofrecer a Fichte es ésta: distinguir entre la filosofía y la persona. A pesar de considerar atea su doctrina, al igual que la de Spinoza, Jacobi confiesa que no lo tiene personalmente por un impío. Fichte ha sabido elevarse con el espíritu sobre la naturaleza y ha visto a Dios cara a cara. Pero su filosofía es, sin embargo, atea "según el juicio correcto de la razón natural, que llama absurdo a un dios que no sea personal, un dios que no es." (JaF 45-46)

Al identificar, en su escrito Sobre el fundamento de nuestra creencia en un gobierno divino del mundo, a Dios con el orden moral del mundo, Fichte está siendo, entonces, coherente con el punto de vista que ha decidido adoptar: el de una filosofía pura y acabada en sí misma. La negación de la existencia de un Dios trascendente y personal y el establecimiento de una unidad artificial que reemplaza esa dualidad originaria del entendimiento natural responde, pues, a la exigencia de la razón especulativa, que intenta reducirlo todo a un único principio. Si Fichte hubiera evitado este punto de vista, habría logrado escuchar a su corazón y, en vez de postular a un falso dios, habría 
reconocido al Dios verdadero. De modo que Jacobi afirma que es su filosofía la que debe ser reprendida, pero no su persona. ${ }^{18}$

\section{La apuesta de Jacobi}

31 Frente a la filosofía que conduce al abismo de la negación de la divinidad y del mundo, Jacobi propone abrazar el no-saber. La filosofía no puede ser refutada; tampoco se la puede depurar. Como Pascal, Jacobi considera que la única alternativa es burlarse de ella. (cf. JaF 38) Reírse de la pretensión de explicarlo todo mediante razones es ya haberla abandonado. Quien ríe ha saltado.

Se revela aquí la conclusión del largo argumento que Jacobi construye en su Carta a Fichte: se trata de una elección, la única que debe hacer el hombre: "la nada o un Dios". (JaF 49) La identificación de los proyectos de Fichte y de Spinoza, la referencia a Fichte como el Mesías de la razón pura, es un paso necesario en este argumento, pues Jacobi precisa establecer que no hay una tercera alternativa. Optar por la razón especulativa ser spinozista o convertirse a la Doctrina de la ciencia - significa negar al único Dios que lo es verdaderamente, abrazar el nihilismo, condenarse a la desesperación. Y, como queda claro a partir de la conversación que muchos años antes Jacobi había mantenido con Lessing, (véase JW IV.1, 48) no hay argumentos para convencer a alguien de adoptar uno $u$ otro modo de vida.

Parecería, pues, que al menos en este punto, Jacobi podría haber reconocido una coincidencia con Fichte. La postura filosófica que cada uno elige depende de qué clase de hombre se es, había dicho Fichte. (cf. GA I, 4, 195 EE \$5) Ser filósofo o abrazar la nofilosofía depende del mundo en el que cada uno quiera vivir, podría haber dicho Jacobi; depende, en definitiva, de lo que cada uno pueda soportar.

La interpretación de Jacobi, que ve en Fichte al heredero del spinozismo, al Cristo de la razón especulativa, a la nueva cumbre del racionalismo, tiene un carácter provocativo y debe ser considerada en el contexto de la ya antigua y aún violenta batalla de Jacobi contra la Ilustración. Sin embargo, podría pensarse que el propio Fichte, al situar a Spinoza como el máximo representante del dogmatismo -la única alternativa a su propio sistema- habría proveído a su detractor suficientes elementos para considerar la Doctrina de la ciencia como un spinozismo invertido. ${ }^{19}$

En efecto, algunos han visto en el sistema fichteano, la transformación de la sustancia de Spinoza en un Yo absoluto, puramente activo. ${ }^{20} \mathrm{~J}-\mathrm{M}$. Vaysse, por ejemplo, señala que el panteísmo ontológico de Spinoza, se transforma en Fichte en un panteísmo práctico: mientras que en el sistema spinoziano la natura naturans se expresa necesariamente en la natura naturata, el Yo absoluto fichteano debe realizarse en el ámbito de la práctica. ${ }^{21}$ El Dios de Spinoza, idéntico con la naturaleza y sus leyes necesarias, se transforma, en Fichte, en el orden moral del mundo. Y, aunque en Spinoza el orden natural sea un infinito actualizado y en Fichte el orden moral sea siempre un infinito a realizar, ambos comparten el principio de la inmanencia y niegan al unísono la idea de una divinidad trascendente, que imponga sus leyes eternas a la naturaleza, que regule la obediencia de los hombres. Esto era suficiente para que Jacobi no pudiera aceptar sus puntos de vista, por mucha simpatía que le inspiraran sus personas.

La caracterización de la Doctrina de la ciencia como una forma del spinozismo permite a Jacobi concentrarse en el aspecto de Fichte que él no puede aceptar: la pretensión de 
explicar lo Absoluto. Si hubo en Fichte un comienzo intuitivo, ligado a la vida y a la conciencia común, Jacobi considera que éste se pierde en su esfuerzo por sistematizarlo, por diseccionarlo y estudiar sus componentes. Al hacer esto, Fichte ha tomado el camino de la filosofía, el mismo de Spinoza y de todos los hombres que sólo atienden al impulso por conocerlo todo. Al adoptar este punto de vista, el de la abstracción, Fichte se ha apartado de la vida y ha caído en el error que él quería evitar. Como Spinoza, Fichte ha reducido toda la realidad a un único principio. A diferencia de Spinoza, Fichte ha visto que sólo la pura actividad pensante de un Yo puede ser verdadero fundamento. Su virtud ha sido dar ese paso final: el abismo ya no es en Fichte una consecuencia del sistema, sino que esa nada es la materia de la que está hecho su Yo absoluto, un Yo absolutamente vacío.

Fichte debió haberse sentido profundamente decepcionado por la incapacidad de Jacobi de encontrar en su filosofía las grandes coincidencias que él había visto entre ellos. Jacobi le reprochaba justamente lo que Fichte había creído aprender de él, le reprochaba haber querido transformar la vida en ciencia y, por lo tanto, haber hecho de Dios un concepto muerto. "Mi filosofía tiene su esencia en el no-saber tanto como la de Jacobi", protesta Fichte en una carta a Reinhold del del 8 de enero de 1800. (GA III, 4, 181) Según Fichte, Jacobi parece haber olvidado la parte práctica de su sistema. No vio en qué medida la Doctrina de la Ciencia era el sistema de la libertad, una meditación acerca de la vida.

Digamos, para terminar, que aún si existen diferencias que distancian a Jacobi de Fichte, evidentemente se dan algunas coincidencias importantes entre ambos. Una coincidencia concierne a su actitud respecto del spinozismo. Ambos le otorgan a Spinoza un lugar similar en la construcción de sus propias posiciones: Fichte lo considera como la formulación más coherente del dogmatismo frente a la cual construye su Doctrina de la ciencia; Jacobi lo contempla con horror, por encarnar todos los males de una razón desmedida, pero a su vez con admiración, pues la valentía de Spinoza, que lleva la razón a sus límites, pone en evidencia la imposibilidad de permanecer en su sistema, la necesidad de dar el salto al ámbito de la fe, de la vida.

Pero más allá de esto, que podría considerarse una coincidencia negativa -puesto que concuerdan en que ninguno quiere ser spinozista-, Fichte y Jacobi coinciden, de hecho, en algo mucho más fundamental: ambos destronan a la razón teórica y colocan en su lugar, como su fundamento, una capacidad diferente, no discursiva, no conceptual y que, por tanto, puede ser caracterizada como no-saber..$^{22}$ Aunque por diferentes motivos, ambos se enfrentan así al racionalismo de la primera Ilustración alemana. "Jacobi parece confundirme con Mendelssohn o con algún otro de sus semejantes, que quieren introducir por medio del razonamiento toda la religión en los hombres. ¿No se da cuenta de que yo odio tanto como él e incluso más [que él] la obra de los Nicolaitas?", (GA, III, 4, 181) escribe Fichte a Reinhold.

41 Jacobi no pudo, o no quiso, ver esta coincidencia entre ellos. Fichte seguirá esforzándose en ser reconocido como discípulo por él, su segundo maestro. En los años siguientes, explicitará una y otra vez la supremacía de la razón práctica por sobre la razón teórica y mostrará en qué medida la Doctrina de la Ciencia se funda en un obrar y no en un saber. 


\section{NOTAS}

1. Citaré el texto de Jacobi Jacobi an Fichte (de aquí en más JaF) según la paginación de su primera edición (Hamburgo 1799). La Carta se publicó también en el tercer volúmen de Friedrich Heinrich Jacobis Werke, Köppen y Roth (ed.), Leipzig 1812-1825 (reimp.: Darmstadt 1968), 6 tomos (de aquí en más JW) y en el segundo volúmen de la reciente edición crítica: Jacobis Werke, K. Hammacher y W. Jaeschke (eds.), Hamburg 1998 ss. Además de la traducción al español de V. Serrano, contamos con la traducción de J. L. Villacañas, incluida en F. H. Jacobi, Cartas a Mendelssohn. Carta a Hume. Carta a Fichte. Barcelona 1996. Cito la correspondencia de Jacobi según la edición Friedrich Heinrich Jacobi's Auserlesener Briefwechsel. Leipzig 1827 (de aquí en más JABr).

2. Respecto de la compleja relación entre Fichte y Jacobi véase R. LAUTH, "Fichtes Verhältnis zu Jacobi unter besonderer Brücksichtigung der Rolle Friedrich Schlegels in dieser Sache", en: K. Hammacher (ed.) Friedrich Heinrich Jacobi. Philosoph und Literat der Goethezeit. Frankfurt am Main 1971. Véase también G. DI GIOVANNI "From Jacobi's Philosophical Novel to Fichte's Idealism: Some Comments on the 1798-99 "Atheism Dispute"', en: Journal of the History of Philosophy, 27, (1) 1989: 75-100.

3. Jacobi publicó en 1792 su Antología de cartas de Allwill (Allwills Briefsammlung), versión definitiva de una novela que había aparecido por entregas entre 1775 y 1776. Bajo la forma literaria de la novela psicológica, esta obra expone las pretensiones filosóficas de Jacobi. (cf. JW I, 6) En la versión impresa de su Carta a Fichte, Jacobi incluye en el Añadido III el prefacio a esta novela. (cf. JaF 95 ss.)

4. David Hume über den Glauben oder Idealismus und Realismus, Apéndice.

5. Carta de Jacobi a W.v. Humboldt del 2 de septiembre de 1794.

6. Cf. I. RADRIZZANI, “Jacobis Auseinandersetzung mit Fichte in den Denkbüchern", en: F-S 14, 1998: 44. Véase también P. SCHNEIDER, Die “Denkbücher" F.H. Jacobis, Stuttgart-Bad Cannstatt 1986.

7. Cf. I. RADRIZZANI, "Jacobis Auseinandersetzung mit Fichte in den Denkbüchern", en: F-S 14, 1998: 45.

8. Ibíd.

9. Véase K. WIEDMANN, Die Bibliothek Friedrich Heinrich Jacobi. Ein Katalog, Stuttgart-Bad Cannstatt 1989.

10. Cf. A. IAVOCACCI, Idealismo e Nichilismo. La Lettera di Jacobi a Fichte. Padova 1992: 84.

11. Cf. ibíd. 90 y 91

12. Acerca de las consecuencias del Pantheismusstreit en el desarrollo de la Ilustración Alemana, véase F. BEISER, The Fate of Reason. German Philosophy from Kant to Fichte. Cambridge-MassachusettsLondon 1987: 44 ss. Véase también H. TIMM, Gott und die Freiheit. Studien zur Religionsphilosophie der Goethezeit.I: Die Spinozarenaissance. Frankfurt am Main 1974.

13. El uso que hace aquí Jacobi del término 'materialismo' podría remitirse, tal como sugiere G. Zöller, a la Primera introducción a la doctrina de la ciencia.. En efecto, Fichte emplea allí este término en el mismo sentido, pues lo utiliza para reemplazar la denominación 'dogmatismo'. (cf. GA I, 4, 197 ss. EE §6) Además, Zöller muestra que la oposición entre materialismo (o dogmatismo) e idealismo no es un aporte original de Jacobi, ella se halla en escritos de Schelling y Fichte publicados anteriormente a JaF. Ya en 1795 Schelling había desarrollado esta oposición en sus Philosophische Briefe über Dogmatismus und Kriticismus, la cual influyó en la distinción que hace Fichte en EE entre Idealismo y Dogmatismo o Materialismo. (cf. GA I, 4, 188-208 EE §§ 3-8) (véase G. zÖLLER, "Fichte als Spinoza, Spinoza als Fichte", en W. Jaeschke y B. Sandkaulen (eds.), Friedrich Heinrich Jacobi. Ein Wendepunkt der geistigen Bildung der Zeit. Hamburg 2004: 45) 
14. Hay que tener en cuenta que Jacobi utiliza aquí el vocabulario medieval. La cara superior sería el pensamiento o "ser objetivo" (ser en la representación) y la inferior el ser o "ser subjetivo o formal” (ser trascendente de la representación, independiente de la representación).

15. Véase especialmente el Apéndice VII añadido a la segunda edición de Über die Lehre des Spinoza in Briefen an den Herrn Moses Mendelssohn.

16. Ver W. JAESCHKE, "Der Messias der spekulativen Vernunft", en: Kritisches Jahrbuch der Philosophie, 4 (1999): 144.

17. Jacobi había esbozado esta dialéctica entre el yo y el tú en Über die Lehre des Spinoza in Briefen an den Herrn Moses Mendelssohn y la desarrolla en profundidad en David Hume über den Glauben oder Idealismus und Realismus.

18. Jaeschke indica que esta defensa es problemática, en la medida en que sólo es exitosa a costa de la filosofía de Fichte pero también de su competencia en cuanto filósofo. En efecto, si la negación de Dios es sólo un error, un movimiento de los labios, entonces toda su filosofía lo es también (cf. W. JAESCHKE op. cit. 153-4).

19. R. Lauth sostiene que Jacobi llega a esta ocurrencia influenciado por Weishuhn, Hölderlin y, en particular, de Schelling, quienes ya en octubre de 1794 y a principios de 1795 habían caracterizado la posición fichteana como spinozista o como un spinozismo modificado y que, de hecho, la caracterización es incorrecta (cf. R. LAUTH, "Das Fehlverständnis der Wissenschaftslehre als subjektiver Spinozismus", en su: Vernünftige Durchdringung der Wirklichkeit. Fichte und sein Umkreis. Neuried 1994: 29-54)

20. Véase por ejemplo X. LÉON, Fichte et son temps. Paris 1922; G. DI GIOVANNI, ob. cit. y J.-M. VAYSSE, Totalité et Subjectivité, Spinoza dans l'Ideallisme Allemand. Paris 1994.

21. Véase J.-M. VAYSSE op. cit. 84.

22. Di Giovanni sostiene que, reaccionando contra el racionalismo de la vieja escuela - aunque por motivos diferentes -, ambos atribuyen a la razón un rol nuevo, más artístico, histórico, concreto y que, en este sentido, ambos responden a las exigencias del romanticismo. (cf. G. DI GIOVANNI, op. cit. 77)

\section{RESÚMENES}

This article deals with F. H. Jacobi's criticism of Fichte's thought, exposed it in his Briefe an Fichte, of 1799. In order to offer the context of the discussion between them, we analyze, in the first place, the connection that existed between both philosophers before the Atheismusstreit. Then, we expose Jacobi's interpretation of the Wissenschaftslehre as the most accomplished system in the history of rationalist philosophy -for which Fichte is designated as the Messiah of speculative reason-. To conclude, we analyze briefly Fichte's reaction to these criticisms. The article shows that, just as Fichte claims, and contrary to what Jacobi affirms, there are some coincidences between them concerning their philosophical views. They agree in their appraisal of Spinozism; but fundamentally they concur in that they both dethrone speculative reason and set above it a not demonstrative, not discursive power that can be in both cases considered as "nicht-Wissen". 
ÍNDICE

Keywords: Jacobi, rationalism, atheism

AUTOR

MARÍA JIMENA SOLÉ

Buenos Aires 